\title{
Maximal and minimal point theorems and Caristi's fixed point theorem
}

\author{
Zhilong Li and Shujun Jiang
}

* Correspondence: Izl771218@sina. com

Department of Mathematics, Jiangxi University of Finance and Economics, Nanchang, Jiangxi 330013, China

\begin{abstract}
This study is concerned with the existence of fixed points of Caristi-type mappings motivated by a problem stated by Kirk. First, several existence theorems of maximal and minimal points are established. By using them, some generalized Caristi's fixed point theorems are proved, which improve Caristi's fixed point theorem and the results in the studies of Jachymski, Feng and Liu, Khamsi, and Li.
\end{abstract}

MSC 2010: 06A06; 47H10.

Keywords: maximal and minimal point, Caristi's fixed point theorem, Caristi-type mapping, partial order

\section{Introduction}

In the past decades, Caristi's fixed point theorem has been generalized and extended in several directions, and the proofs given for Caristi's result varied and used different techniques, we refer the readers to [1-15].

Recall that $T: X \rightarrow X$ is said to be a Caristi-type mapping [14] provided that there exists a function $\eta:[0,+\infty) \rightarrow[0,+\infty)$ and a function $\phi: X \rightarrow(-\infty,+\infty)$ such that

$$
\eta(d(x, T x)) \leq \varphi(x)-\varphi(T x), \quad \forall x \in X,
$$

where $(X, d)$ is a complete metric space. Let $\leqslant$ be a relationship defined on $X$ as follows

$$
x \preccurlyeq y \Leftrightarrow \eta(d(x, y)) \leq \varphi(x)-\varphi(y), \quad \forall x, y \in X .
$$

Clearly, $x \leqslant T x$ for each $x \in X$ provided that $T$ is a Caristi-type mapping. Therefore, the existence of fixed points of Caristi-type mappings is equivalent to the existence of maximal point of $(X, \preccurlyeq)$. Assume that $\eta$ is a continuous, nondecreasing, and subadditive function with $\eta^{-1}(\{0\})=\{0\}$, then the relationship defined by (1) is a partial order on $X$. Feng and Liu [12] proved each Caristi-type mapping has a fixed point by investigating the existence of maximal point of $(X, \preccurlyeq)$ provided that $\phi$ is lower semicontinuous and bounded below. The additivity of $\eta$ appearing in [12] guarantees that the relationship $\leqslant$ defined by (1) is a partial order on $X$. However, if $\eta$ is not subadditive, then the relationship $\leqslant$ defined by (1) may not be a partial order on $X$, and consequently the method used there becomes invalid. Recently, Khamsi [13] removed the additivity of $\eta$ by introducing a partial order on $Q$ as follows

(c) $2011 \mathrm{Li}$ and Jiang; licensee Springer. This is an Open Access article distributed under the terms of the Creative Commons Attribution License (http://creativecommons.org/licenses/by/2.0), which permits unrestricted use, distribution, and reproduction in any medium, provided the original work is properly cited. 


$$
x \preccurlyeq * y \Leftrightarrow c d(x, y) \leq \varphi(x)-\varphi(y), \quad \forall x, y \in Q,
$$

where $Q=\left\{x \in X: \varphi(x) \leq \inf _{t \in X} \varphi(t)+\varepsilon\right\}$ for some $\varepsilon>0$. Assume that $\phi$ is lower semicontinuous and bounded below, $\eta$ is continuous and nondecreasing, and there exists $\delta$ $>0$ and $c>0$ such that $\eta(t) \geq c t$ for each $t \in[0, \delta]$. He showed that $\left(Q, \varsigma^{*}\right)$ has a maximal point which is exactly the maximal point of $(X, \preccurlyeq)$ and hence each Caristi-type mapping has a fixed point. Very recently, the results of $[9,12,13]$ were improved by Li [14] in which the continuity, subadditivity and nondecreasing property of $\eta$ are removed at the expense that

$(H)$ there exists $c>0$ and $\varepsilon>0$ such that $\eta(t) \geq c t$ for each

$$
t \in\{t \geq 0: \eta(t) \leq \varepsilon\}
$$

From [14, Theorem 2 and Remark 2] we know that the assumptions made on $\eta$ in $[12,13]$ force that $(H)$ is satisfied. In other words, $(H)$ is necessarily assumed in [12-14]. Meanwhile, $\phi$ is always assumed to be lower semicontinuous there.

In this study, we shall show how the condition $(H)$ and the lower semicontinuity of $\phi$ could be removed. We first proved several existence theorems of maximal and minimal points. By using them, we obtained some fixed point theorems of Caristi-type mappings in a partially ordered complete metric space without the lower semicontinuity of $\phi$ and the condition $(H)$.

\section{Maximal and minimal point theorems}

For the sake of convenience, we in this section make the following assumptions:

$\left(H_{1}\right)$ there exists a bounded below function $\phi: X \rightarrow(-\infty,+\infty)$ and a function $\eta:[0$, $+\infty) \rightarrow[0,+\infty)$ with $\eta^{-1}(\{0\})=\{0\}$ such that

$$
\eta(d(x, y)) \leq \varphi(x)-\varphi(y)
$$

for each $x, y \in X$ with $x \leqslant y$;

$\left(H_{2}\right)$ for any increasing sequence $\left\{x_{n}\right\}_{n \geq 1} \subset X$, if there exists some $x \in X$ such that $x_{n}$ $\rightarrow x$ as $n \rightarrow \infty$, then $x_{n} \leqslant x$ for each $n \geq 1$;

$\left(H_{3}\right)$ for each $x \in X$, the set $\{y \in X: x \preccurlyeq y\}$ is closed;

$\left(H_{4}\right) \eta$ is nondecreasing;

$\left(H_{5}\right) \eta$ is continuous and $\liminf _{t \rightarrow+\infty} \eta(t)>0$;

$\left(H_{6}\right)$ there exists a bounded above function $\phi: X \rightarrow(-\infty,+\infty)$ and a function $\eta:[0$, $+\infty) \rightarrow[0,+\infty)$ with $\eta^{-1}(\{0\})=\{0\}$ such that (2) holds for each $x, y \in X$ with $x \leqslant y$;

$\left(H_{7}\right)$ for any decreasing sequence $\left\{x_{n}\right\}_{n \geq 1} \subset X$, if there exists some $x \in X$ such that $x_{n}$ $\rightarrow x$ as $n \rightarrow \infty$, then $x \leqslant x_{n}$ for each $n \geq 1$;

$\left(H_{8}\right)$ for each $x \in X$, the set $\{y \in X: y \leqslant x\}$ is closed.

Recall that a point $x^{*} \in X$ is said to be a maximal (resp. minimal) point of $(X, \preccurlyeq)$ provided that $x=x^{*}$ for each $x \in X$ with $x^{*} \leqslant x$ (resp. $\left.x \leqslant x^{*}\right)$.

Theorem 1. Let $(X, d, \preccurlyeq)$ be a partially ordered complete metric space. If $\left(H_{1}\right)$ and $\left(H_{2}\right)$ hold, and $\left(H_{4}\right)$ or $\left(H_{5}\right)$ is satisfied, then $(X, \preccurlyeq)$ has a maximal point.

Proof. Case 1. $\left(H_{4}\right)$ is satisfied. Let $\left\{x_{\alpha}\right\}_{\alpha \in \Gamma} \subset F$ be an increasing chain with respect to the partial order $\preccurlyeq$. From (2) we find that $\left\{\phi\left(x_{\alpha}\right)\right\}_{\alpha \in \Gamma}$ is a decreasing net of reals, where $\Gamma$ is a directed set. Since $\phi$ is bounded below, then $\underset{\alpha \in \Gamma}{\inf } \varphi\left(x_{\alpha}\right)$ is meaningful. Let 
$\left\{\alpha_{n}\right\}$ be an increasing sequence of elements from $\Gamma$ such that

$$
\lim _{n \rightarrow \infty} \varphi\left(x_{\alpha_{n}}\right)=\inf _{\alpha \in \Gamma} \varphi\left(x_{\alpha}\right) .
$$

We claim that $\left\{x_{\alpha_{n}}\right\}_{n \geq 1}$ is a Cauchy sequence. Otherwise, there exists a subsequence $\left\{x_{\alpha_{n_{i}}}\right\}_{i \geq 1} \subset\left\{x_{\alpha_{n}}\right\}_{n \geq 1}$ and $\delta>0$ such that $x_{\alpha_{n_{i}}} \preccurlyeq x_{\alpha_{n_{i+1}}}$ for each $i \geq 1$ and

$$
d\left(x_{\alpha_{n_{i}}}, x_{\alpha_{n_{i+1}}}\right) \geq \delta, \quad \forall i \geq 1 .
$$

By $(4)$ and $\left(H_{4}\right)$, we have

$$
\eta\left(d\left(x_{\alpha_{n_{i}}}, x_{\alpha_{n_{i+1}}}\right)\right) \geq \eta(\delta), \quad \forall i \geq 1 .
$$

Therefore from (2) and (5) we have

$$
\varphi\left(x_{\alpha_{n_{i}}}\right)-\varphi\left(x_{\alpha_{n_{i+1}}}\right) \geq \eta(\delta), \quad \forall i \geq 1,
$$

which indicates that

$$
\varphi\left(x_{\alpha_{n_{i+1}}}\right) \leq \varphi\left(x_{\alpha_{n_{1}}}\right)-i \eta(\delta), \quad \forall i \geq 1 .
$$

Let $i \rightarrow \infty$ in (6), by (3) and $\eta^{-1}(\{0\})=\{0\}$ we have

$$
\inf _{\alpha \in \Gamma} \varphi\left(x_{\alpha}\right)=\lim _{i \rightarrow \infty} \varphi\left(x_{\alpha_{n_{i}}}\right) \leq-\infty .
$$

This is a contradiction, and consequently, $\left\{x_{\alpha_{n}}\right\}_{n \geq 1}$ is a Cauchy sequence.

Therefore by the completeness of $X$, there exists $x \in X$ such that $x_{\alpha_{n}} \rightarrow x$ as $n \rightarrow \infty$. Moreover, $\left(H_{2}\right)$ forces that

$$
x_{\alpha_{n}} \preccurlyeq x, \quad \forall n \geq 1 .
$$

In the following, we show that $\left\{x_{\alpha}\right\}_{\alpha \in \Gamma}$ has an upper bound. In fact, for each $\alpha \in \Gamma$, if there exists some $n \geq 1$ such that $x_{\alpha} \preccurlyeq x_{\alpha_{n}}$, by (7) we get $x_{\alpha} \preccurlyeq x_{\alpha_{n}} \preccurlyeq x$, i.e., $x$ is an upper bound of $\left\{x_{\alpha}\right\}_{\alpha \in \Gamma}$. Otherwise, there exists some $\beta \in \Gamma$ such that $x_{\alpha_{n}} \preccurlyeq x_{\beta}$ for each $n \geq 1$. From (2) we find that $\varphi\left(x_{\beta}\right) \leq \varphi\left(x_{\alpha_{n}}\right)$ for each $n \geq 1$. This together with (3) implies that $\varphi\left(x_{\beta}\right)=\inf _{\alpha \in \Gamma} \varphi\left(x_{\alpha}\right)$ and hence $\phi\left(x_{\beta}\right) \leq \phi\left(x_{\alpha}\right)$ for each $\alpha \in \Gamma$. Note that $\left\{\phi\left(x_{\alpha}\right)\right\}_{\alpha \in \Gamma}$ is a decreasing chain, then we have $\beta \geq \alpha$ for each $\alpha \in \Gamma$. Since $\left\{x_{\alpha}\right\}_{\alpha \in \Gamma}$ is an increasing chain, then $x_{\alpha} \leqslant x_{\beta}$ for each $\alpha \in \Gamma$. This shows that $x_{\beta}$ is an upper bound of $\left\{x_{\alpha}\right\}_{\alpha \in \Gamma}$.

By Zorn's lemma we know that $(X, \preccurlyeq)$ has a maximal point $x^{*}$, i.e., if there exists $x \in$ $X$ such that $x^{*} \leqslant x$, we must have $x=x^{*}$.

Case 2. $\left(H_{5}\right)$ is satisfied. By $\liminf _{t \rightarrow+\infty} \eta(t)>0$, there exists $l>\delta$ and $c_{1}>0$ such that

$$
\eta(t) \geq c_{1}, \quad \forall t \geq l
$$

Since $\eta$ is continuous and $\eta^{-1}(\{0\})=\{0\}$, then $c_{2}=\min _{t \in[\delta, l]} \eta(t)>0$. Let $c=\min \left\{c_{1}, c_{2}\right\}$, then by (4) we have

$$
\eta\left(d\left(x_{\alpha_{n_{i}}}, x_{\alpha_{n_{i+1}}}\right)\right) \geq c, \quad \forall i \geq 1 .
$$


In analogy to Case 1 , we know that $(X, \preccurlyeq)$ has a maximal point. The proof is complete.

Theorem 2. Let $(X, d, \preccurlyeq)$ be a partially ordered complete metric space. If $\left(H_{6}\right)$ and $\left(H_{7}\right)$ hold, and $\left(H_{4}\right)$ or $\left(H_{5}\right)$ is satisfied, then $(X, \preccurlyeq)$ has a minimal point.

Proof. Let $\preccurlyeq_{1}$ be an inverse partial order of $\leqslant$, i.e., $x \leqslant y \Leftrightarrow y \leqslant_{1} x$ for each $x, y \in X$. Let $\varphi(x)=-\phi(x)$. Then, $\varphi$ is bounded below since $\phi$ is bounded above, and hence from $\left(H_{6}\right)$ and $\left(H_{7}\right)$ we find that both $\left(H_{1}\right)$ and $\left(H_{2}\right)$ hold for $\left(X, d, \preccurlyeq_{1}\right)$ and $\varphi$. Finally, Theorem 2 forces that $\left(X, \preccurlyeq_{1}\right)$ has a maximal point which is also the minimal point of $(X$, $\preccurlyeq)$. The proof is complete.

Theorem 3. Let $(X, d, \preccurlyeq)$ be a partially ordered complete metric space. If $\left(H_{1}\right)$ and $\left(H_{3}\right)$ hold, and $\left(H_{4}\right)$ or $\left(H_{5}\right)$ is satisfied, then $(X, \preccurlyeq)$ has a maximal point.

Proof. Following the proof of Theorem 1, we only need to show that (7) holds. In fact, for arbitrarily given $n_{0} \geq 1,\left\{y \in X: x_{\alpha_{n_{0}}} \preccurlyeq y\right\}$ is closed by $\left(H_{3}\right)$. From (2) we know that $x_{\alpha_{n_{0}}} \preccurlyeq x_{\alpha_{n}}$ as $n \geq n_{0}$ and hence $x_{\alpha_{n}} \in\left\{y \in X: x_{\alpha_{n_{0}}} \preccurlyeq y\right\}$ for all $n \geq n_{0}$. Therefore, we have $x \in\left\{y \in X: x_{\alpha_{n_{0}}} \preccurlyeq \gamma\right\}$, i.e., $x_{\alpha_{n_{0}}} \preccurlyeq x$. Finally, the arbitrary property of $n_{0}$ implies that (7) holds. The proof is complete.

Similarly, we have the following result.

Theorem 4. Let $(X, d, \preccurlyeq)$ be a partially ordered complete metric space. If $\left(H_{6}\right)$ and $\left(H_{8}\right)$ hold, and $\left(H_{4}\right)$ or $\left(H_{5}\right)$ is satisfied, then $(X, \preccurlyeq)$ has a minimal point.

\section{Caristi's fixed point theorem}

Theorem 5. Let $(X, d, \preccurlyeq)$ be a partially ordered complete metric space and $T: X \rightarrow X$. Suppose that $\left(H_{1}\right)$ holds, and $\left(H_{2}\right)$ or $\left(H_{3}\right)$ is satisfied. If $\left(H_{4}\right)$ or $\left(H_{5}\right)$ is satisfied, then $T$ has a fixed point provided that $x \leqslant$ Tx for each $x \in X$.

Proof. From Theorems 1 and 3, we know that $(X, \preccurlyeq)$ has a maximal point. Let $x^{*}$ be a maximal point of $(X, \preccurlyeq)$, then $x^{*} \leqslant T x^{*}$. The maximality of $x^{*}$ forces $x^{*}=T x^{*}$, i.e., $x^{*}$ is a fixed point of $T$. The proof is complete.

Theorem 6. Let $(X, d, \preccurlyeq)$ be a partially ordered complete metric space and $T: X \rightarrow$ $X$. Suppose that $\left(H_{6}\right)$ holds, and $\left(H_{7}\right)$ or $\left(H_{8}\right)$ is satisfied. If $\left(H_{4}\right)$ or $\left(H_{5}\right)$ is satisfied, then $T$ has a fixed point provided that $T x \leqslant x$ for each $x \in X$.

Proof. From Theorems 2 and 4 , we know that $(X, \preccurlyeq)$ has a minimal point. Let $x^{* *}$ be a minimal point of $(X, \preccurlyeq)$, then $T x^{*} \leqslant x^{*}$. The minimality of $x^{*}$ forces $x^{*}=T x^{*}$, i.e., $x^{*}$ is a fixed point of $T$. The proof is complete.

Remark 1. The lower semicontinuity of $\phi$ and $(H)$ necessarily assumed in [9,12-14]are no longer necessary for Theorems 5 and 6. In what follows we shall show that Theorem 5 implies Caristi's fixed point theorem.

The following lemma shows that there does exist some partial order $\leqslant$ on $X$ such that $\left(H_{3}\right)$ is satisfied.

Lemma 1. Let $(X, d)$ be a metric space and the relationship $\leqslant$ defined by (1) be a partial order on $X$. If $\eta:[0,+\infty) \rightarrow[0,+\infty)$ is continuous and $\phi: X \rightarrow(-\infty,+\infty)$ is lower semicontinuous, then $\left(H_{3}\right)$ holds.

Proof. For arbitrary $x \in X$, let $\left\{x_{n}\right\}_{n \geq 1} \subset\{y \in X: x \leqslant y\}$ be a sequence such that $x_{n} \rightarrow$ $x^{*}$ as $n \rightarrow \infty$ for some $x^{*} \in X$. From (1) we have

$$
\eta\left(d\left(x, x_{n}\right)\right) \leq \varphi(x)-\varphi\left(x_{n}\right) .
$$


Let $n \rightarrow \infty$ in (8), then

$$
\underset{n \rightarrow \infty}{\limsup } \eta\left(d\left(x, x_{n}\right)\right) \leq \limsup _{n \rightarrow \infty}\left(\varphi(x)-\varphi\left(x_{n}\right)\right) \leq \varphi(x)-\liminf _{n \rightarrow \infty} \varphi\left(x_{n}\right) .
$$

Moreover, by the continuity of $\eta$ and the lower semicontinuity of $\phi$ we get

$$
\eta\left(d\left(x, x^{*}\right)\right) \leq \varphi(x)-\varphi\left(x^{*}\right)
$$

which implies that $x \leqslant x^{*}$, i.e., $x^{*} \in\{y \in X: x \leqslant y\}$. Therefore, $\{y \in X: x \leqslant y\}$ is closed for each $x \in X$. The proof is complete.

By Theorem 5 and Lemma 1 we have the following result.

Corollary 1. Let $(X, d)$ be a complete metric space and the relationship $\leqslant$ defined by (1) be a partial order on $X$. Let $T: X \rightarrow X$ be a Caristi-type mapping and $\phi$ be a lower semicontinuous and bounded below function. If $\eta$ is a continuous function with $\eta^{-1}(\{0\})$ $=\{0\}$, and $\left(H_{4}\right)$ or $\liminf _{t \rightarrow+\infty} \eta(t)>0$ is satisfied, then $T$ has a fixed point.

It is clear that the relationship defined by (1) is a partial order on $X$ for when $\eta(t)=$ $t$. Then, we obtain the famous Caristi's fixed point theorem by Corollary 1 .

Corollary 2 (Caristi's fixed point theorem). Let $(X, d)$ be a complete metric space and $T: X \rightarrow X$ be a Caristi-type mapping with $\eta(t)=t$. If $\phi$ is lower semicontinuous and bounded below, then $T$ has a fixed point.

Remark 2. From [14, Remarks 1 and 2] we find that [14, Theorem 1] includes the results appearing in $[3,4,9,12,13]$. Note that [14, Theorem 1] is proved by Caristi's fixed point theorem, then the results of [9,12-14] are equivalent to Caristi's fixed point theorem. Therefore, all the results of [3,4,9,12-14]could be obtained by Theorem 5. Contrarily, Theorem 5 could not be derived from Caristi's fixed point theorem. Hence, Theorem 5 indeed improve Caristi's fixed point theorem.

Example 1. Let $X=\{0\} \cup\left\{\frac{1}{n}: n=2,3, \ldots\right\}$ with the usual metric $d(x, y)=|x-y|$ and the partial order $\preccurlyeq$ as follows

$$
x \preccurlyeq y \Leftrightarrow y \leq x .
$$

Let $\phi(x)=x^{2}$ and

$$
T x= \begin{cases}0, & x=0, \\ \frac{1}{n+1}, & x=\frac{1}{n}, n=2,3, \ldots .\end{cases}
$$

Clearly, $(X, d)$ is a complete metric space, $\left(H_{2}\right)$ is satisfied, and $\phi$ is bounded below. For each $x \in X$, we have $x \geq T x$ and hence $x \leqslant T x$. Let $\eta(t)=t^{2}$. Then $\eta^{-1}(\{0\})=\{0\}$, $\left(H_{4}\right)$ and $\left(H_{5}\right)$ are satisfied. Clearly, (2) holds for each $x, y \in X$ with $x=y$. For each $x$, $y \in X$ with $x \leqslant y$ and $x \neq y$, we have two possible cases.

Case 1. When $x=\frac{1}{n}, n \geq 2$ and $y=0$, we have

$$
\eta(d(x, y))=\frac{1}{n^{2}}=\varphi(x)-\varphi(y)
$$

Case 2. When $x=\frac{1}{n}, n \geq 2$ and $y=\frac{1}{m}, m>n$, we have

$$
\eta(d(x, y))=\frac{(m-n)^{2}}{m^{2} n^{2}}<\frac{m^{2}-n^{2}}{m^{2} n^{2}}=\varphi(x)-\varphi(y) .
$$


Therefore, (2) holds for each $x, y \in X$ with $x \leqslant y$ and hence $\left(H_{1}\right)$ is satisfied. Finally, the existence of fixed point follows from Theorem 5 .

While for each $x=\frac{1}{n}, n \geq 2$, we have $\varphi(x)-\varphi(T x)=\frac{2 n+1}{n^{2}(n+1)^{2}}<\frac{1}{n(n+1)}=d(x, T x)$,

which implies that corresponding to the function $\phi(x)=x^{2}, T$ is not a Caristi-type mapping. Therefore, we can conclude that for some given function $\phi$ and some given mapping $T$, there may exist some function $\eta$ such that all the conditions of Theorem 5 are satisfied even though $T$ may not be a Caristi-type mapping corresponding to the function $\phi$.

\section{Conclusions}

In this article, some new fixed point theorems of Caristi-type mappings have been proved by establishing several maximal and minimal point theorems. As one can see through Remark 2, many recent results could be obtained by Theorem 5, but Theorem 5 could not be derived from Caristi's fixed point theorem. Therefore, the fixed point theorems indeed improve Caristi's fixed point theorem.

\section{Acknowledgements}

This study was supported by the National Natural Science Foundation of China (10701040, 11161022,60964005), the Natural Science Foundation of Jiangxi Province (2009GQS0007), and the Science and Technology Foundation of Jiangxi Educational Department (GJJ11420).

\section{Authors' contributions}

ZL carried out the main part of this article. All authors read and approved the final manuscript.

\section{Competing interests}

The authors declare that they have no competing interests.

Received: 8 August 2011 Accepted: 21 December 2011 Published: 21 December 2011

\section{References}

1. Kirk, WA, Caristi, J: Mapping theorems in metric and Banach spaces. Bull Acad Polon Sci. 23, 891-894 (1975)

2. Kirk, WA: Caristi's fixed-point theorem and metric convexity. Collog Math. 36, 81-86 (1976)

3. Caristi, J: Fixed point theorems for mappings satisfying inwardness conditions. Trans Am Math Soc. 215, $241-251$ (1976)

4. Caristi, J: Fixed point theory and inwardness conditions. In: Lakshmikantham V (ed.) Applied Nonlinear Analysis. pp. 479-483. Academic Press, New York (1979)

5. Brondsted, A: Fixed point and partial orders. Proc Am Math Soc. 60, 365-368 (1976)

6. Downing, D, Kirk, WA: A generalization of Caristi's theorem with applications to nonlinear mapping theory. Pacific J Math. 69, 339-345 (1977)

7. Downing, D, Kirk, WA: Fixed point theorems for set-valued mappings in metric and Banach spaces. Math Japon. 22, 99-112 (1977)

8. Khamsi, MA, Misane, D: Compactness of convexity structures in metrics paces. Math Japon. 41, 321-326 (1995)

9. Jachymski, J: Caristi's fixed point theorem and selection of set-valued contractions. J Math Anal Appl. 227, 55-67 (1998). doi:10.1006/jmaa.1998.6074

10. Bae, JS: Fixed point theorems for weakly contractive multivalued maps. J Math Anal Appl. 284, 690-697 (2003). doi:10.1016/S0022-247X(03)00387-1

11. Suzuki, T: Generalized Caristi's fixed point theorems by Bae and others. J Math Anal Appl. 302, 502-508 (2005). doi:10.1016/j.jmaa.2004.08.019

12. Feng, YQ, Liu, SY: Fixed point theorems for multi-valued contractive mappings and multi-valued Caristi-type mappings. J Math Anal Appl. 317, 103-112 (2006). doi:10.1016/j.jmaa.2005.12.004

13. Khamsi, MA: Remarks on Caristi's fixed point theorem. Nonlinear Anal. 71, 227-231 (2009). doi:10.1016/..na.2008.10.042

14. Li, Z: Remarks on Caristi's fixed point theorem and Kirk's problem. Nonlinear Anal. 73, 3751-3755 (2010). doi:10.1016/j. na.2010.07.048

15. Agarwal, RP, Khamsi, MA: Extension of Caristi's fixed point theorem to vector valued metric space. Nonlinear Anal. 74, 141-145 (2011). doi:10.1016/j.na.2010.08.025 